\title{
Fixed point property and approximation of a class of nonexpansive mappings
}

\author{
Yisheng Song ${ }^{1,2^{*}}$ and Ying Huang ${ }^{1}$
}

"Correspondence:

songyisheng1@gmail.com

'school of Mathematics and Information Science, Henan Normal

University, XinXiang, HeNan 453007,

P.R. China

2Department of Applied

Mathematics, The Hong Kong

Polytechnic University, Hung Hom,

Kowloon, Hong Kong, P.R. China

\begin{abstract}
We introduce the concept of $\psi$-firmly nonexpansive mapping, which includes a firmly nonexpansive mapping as a special case in a uniformly convex Banach space. It is shown that every bounded closed convex subset of a reflexive Banach space has the fixed point property for $\psi$-firmly nonexpansive mappings, an important subclass of nonexpansive mappings. Furthermore, Picard iteration of this class of mappings weakly converges to a fixed point.
\end{abstract}

MSC: 47H06; 47J05; 47J25; 47H10; 47H17

Keywords: $\psi$-firmly nonexpansive mappings; fixed points; reflexive Banach spaces; Picard iteration

\section{Introduction}

Throughout this paper, a Banach space $E$ will be over the real scalar field. We denote its norm by $\|\cdot\|$ and its dual space by $E^{*}$. Let $F(T)=\{x \in E: T x=x\}$, the set of all fixed points for a mapping $T$ and $\mathbb{N}$ denote the set of all positive integer.

Let $K$ be a nonempty bounded closed convex subset of $E$. We say that $K$ has the fixed point property for nonexpansive mapping if for every nonexpansive mapping $T: K \rightarrow K$ (i.e. $\|T x-T y\| \leq\|x-y\| \forall x, y \in K$ ), $K$ contains a fixed point $x^{*}$ of $T$ (i.e. $T x^{*}=x^{*}$ ); $E$ has the fixed point property (FPP for short) if any nonempty bounded closed convex subset of $E$ has the fixed point property for nonexpansive mapping; $E$ has the weak fixed point property (WFPP for short) if any weakly compact convex subset of $E$ has the fixed point property for nonexpansive mapping. For a reflexive Banach space, both properties are obviously the same.

The famous question whether a Banach space has the fixed point property (WFPP) had remained open for a long time [1, 2]. It has been answered in the negative by Sadovski [3] and Alspach [4] who constructed the following examples, respectively.

Example 1.1 (Sadovski [3] or Istrățescu [5, Example 6.3.3]) Let $E=c_{0}$ and $K=\{x \in$ $\left.c_{0} ;\|x\| \leq 1\right\}$. Define $T: K \rightarrow K$ by

$$
T x=\left(1, x_{1}, x_{2}, x_{3}, \ldots\right), \quad \forall x=\left(x_{1}, x_{2}, x_{3}, \ldots\right) \in K .
$$

Example 1.2 (Alspach [4] or Kirk-Sims [6, Example 401]) Let $E=L^{1}[0,1]$ and

$$
K=\left\{x \in L^{1}[0,1] ; 0 \leq x(t) \leq 1 \text { and } \int_{0}^{1} x(t) d t=\frac{1}{2}\right\} .
$$

O2014 Song and Huang; licensee Springer. This is an Open Access article distributed under the terms of the Creative Commons Attribution License (http://creativecommons.org/licenses/by/2.0), which permits unrestricted use, distribution, and reproduction in any medium, provided the original work is properly cited. 
Define $T: K \rightarrow K$ by

$$
(T x)(t)= \begin{cases}\min \{1,2 x(2 t)\} & 0 \leq t \leq \frac{1}{2} \\ \max \{0,2 x(2 t-1)-1\} & \frac{1}{2}<t \leq 1\end{cases}
$$

Then $K$ is bounded, closed, and convex; and $T$ is an isometry $(\|T x-T y\|=\|x-y\| \forall x, y \in$ $K)$ and is fixed point free. For more details, see [3, 4] or [5-7]. Namely, it is proved that $c_{0}$ and $L^{1}[0,1]$ do not have the fixed point property.

The above two examples suggest that to obtain positive results in the problem of the existence of fixed points for nonexpansive mappings, it is necessary to impose some restrictions either on $T$ or on the Banach space $E$. Naturally, the following questions are asked also.

Problem 1.3 Which Banach spaces satisfy the WFPP?

Problem 1.4 Determine a subclass of nonexpansive mappings such that every Banach space satisfies the FPP for this subclass.

Considerable effort in the development of a fixed point theory for nonexpansive mappings, mainly for Problem 1.3, has been done in the last 40 years. A well-known result of Browder [8] asserts that if $E$ is uniformly convex, then $E$ has the weak fixed point property. This theorem was also proved independently by Göhde [9]. At the same time, Kirk [10] established a more general result by showing that if $E$ has normal structure, then $E$ has the weak fixed point property. Normal structure is a geometric property somewhat more general than uniform convexity. In [11], one can see a detailed study of sufficient conditions for this property as well as their permanence properties. It has also been shown [12] that a condition weaker than normal structure is sufficient to guarantee the weak fixed point property (WFPP). In 1981, it has been showed by Maurey [13] that the Hardy space $H^{1}$ and the reflexive subspace of $L^{1}[0,1]$ have the weak fixed point property (WFPP). For other examples of Banach spaces with the weak fixed point property see $[6,7,14]$ and $[15$, 16] for more details.

However, the result about Problem 1.4 is not many. Up to now, a most relevant example is nonlinear isometries in a superreflexive Banach space who is proved by Maurey (see [17, 18] for a proof).

One of our main aims is to give an affirmative answer to Problem 1.4. In other words, we will study fixed point properties of $\psi$-firmly nonexpansive mapping, an important subclass of nonexpansive mappings, on weakly compact convex subsets of a Banach space.

On the other hand, using the Picard iterative method, the well-known Banach Contraction Principle is obtained: Let $(E, d)$ be a complete metric space and $T: E \rightarrow E$ is a contraction (i.e. $d(T x, T y) \leq k d(x, y), \forall x, y \in K$ and some $k \in[0,1)$ ). Then $T$ has a unique fixed point $x^{*}$ and for each $x \in E$, Picard iteration $\left\{T^{n} x\right\}$ strongly converges to $x^{*}$.

It is known for some time that even in a Hilbert space setting, Picard iteration $\left\{T^{n} x\right\}$ of a nonexpansive mapping $T$ need not actually converge to a fixed point. However, for some special nonexpansive mapping (or some nonexpansive mapping who is modified necessarily), the weak convergence of such iteration can be proved. Fox example, in the frame of a uniformly convex Banach space $E$ with a Fréchet differentiable norm, Reich [19] 
showed that if $S=\frac{I+T}{2}$ where $I$ is an identity operator and $T$ is a nonexpansive self-mapping defined on a nonempty bounded closed convex subset $K$ of $E$, then for each $x \in K$, Picard iteration $\left\{S^{n} x\right\}$ weakly converges to a fixed point of $T$; Bruck $[20,21]$ proved that for each $x \in K$, the Cesàro means $T_{n} x=\frac{1}{n+1} \sum_{i=0}^{n} T^{i} x$ of the nonexpansive self-mapping $T$ weakly converges to a fixed point of $T$. This fact was first established by Baillon [22] for the $L^{p}$ spaces $(1<p<\infty)$. Naturally, the following question is put forward.

Problem 1.5 Does there exist a subclass of nonexpansive mappings (not contraction) such that Picard iteration (weakly) converges to a fixed point of such mapping?

Another purpose of ours is to show that Picard iteration of $\psi$-firmly nonexpansive mapping weakly converges to its fixed point. That is, $\psi$-firmly nonexpansive mapping is actually an answer to Problem 1.5 .

We also show that in a uniformly convex space, $\psi$-firmly nonexpansive mapping includes a firmly nonexpansive mapping and the resolvent of an accretive operator as a special case.

\section{Fixed point property for $\psi$-firmly nonexpansive mapping}

The concept of firmly nonexpansive mapping was introduced by Bruck [23]. A mapping $T$ with domain $D(T)$ and range $R(T)$ in Banach space $E$ is said to be firmly nonexpansive if for all $x, y \in D(T)$ the function

$$
\Phi_{x, y}(t)=\|(1-t)(x-y)+t(T x-T y)\|, \quad t \in[0,1]
$$

is non-increasing on $[0,1]$, or equivalently,

$$
\|T x-T y\| \leq\|(1-t)(x-y)+t(T x-T y)\|, \quad x, y \in D(T), t \in[0,1] .
$$

Obviously any firmly nonexpansive mapping is nonexpansive mapping $\left(\Phi_{x, y}(1) \leq \Phi_{x, y}(0)\right)$. The converse is not true (consider the mapping $T x=-x$ in $E$ ). However, there is an interesting observation. For any $u \in D(T)$ and $t \in[0,1)$ consider the $F_{t}$ by $F_{t}=t u+(1-t) T$. Banach Contraction Principle guarantees that $F_{t}$ has a unique fixed point $x_{t}$, i.e.,

$$
x_{t}=F_{t} x_{t}=t u+(1-t) T x_{t} .
$$

Since $x_{t}$ depends on $u$ and $t$, we can define a family mappings $F_{t} u=x_{t}$. It is minor technicality to prove that all mappings $F_{t}$ are firmly nonexpansive. Moreover, for any $t \in[0,1)$, $F\left(F_{t}\right)=F(T)$ (see [7, pp.120-122] for a proof). This shows that the fixed point property for firmly nonexpansive mapping coincides with the fixed point property for nonexpansive mapping.

In a Hilbert space, $T$ is firmly nonexpansive if and only if

$$
\langle T x-T y, x-y\rangle \geq\|T x-T y\|^{2}, \quad x, y \in D(T)
$$

(see [7, pp.127-128] for a proof). Clearly, the inequality (2.2) is equivalent to the following:

$$
\|T x-T y\|^{2} \leq\|x-y\|^{2}-\|(x-T x)-(y-T y)\|^{2}, \quad x, y \in D(T) .
$$


In view of the above one might expect firmly nonexpansive mappings to exhibit better behavior than nonexpansive mappings in general. However, from the point of view of fixed point theory, the restriction is mild. Naturally, Song and Chai [24] introduced the notion of firmly type nonexpansive mapping.

A mapping $T$ is said to be firmly type nonexpansive if for all $x, y \in D(T)$, there exists $k \in(0,+\infty)$ such that

$$
\|T x-T y\|^{2} \leq\|x-y\|^{2}-k\|(x-T x)-(y-T y)\|^{2} .
$$

Obviously, the firmly type nonexpansive mapping contains the firmly nonexpansive mapping and the resolvent of monotone operator as a special case in Hilbert space. For a detailed proof and more examples, see [24, Examples 1-5].

Now we introduce the concept of $\psi$-firmly nonexpansive mapping which includes the firmly type nonexpansive mapping as a special case $\left(\psi(t)=k t^{2}\right)$.

A mapping $T$ is said to be $\psi$-firmly nonexpansive if for all $x, y \in D(T)$, if there exists a continuous strictly increasing function $\psi:[0,+\infty) \rightarrow[0,+\infty)$ with $\psi(0)=0$ such that

$$
\|T x-T y\|^{2} \leq\|x-y\|^{2}-\psi(\|x-T x-(y-T y)\|) .
$$

In order to achieving the objects mentioned in Section 1, solving Problem 1.4, we need the following fact.

Lemma 2.1 ([5, Propositions 9.3.6]) Let $C$ be a weakly compact subset in Banach space $E$ and let $f: C \rightarrow R$ be a weakly lower semi-continuous function. Then the function $f$ attains its minimum on $C$. That is,

$$
\exists x^{*} \in C \text { such that } f\left(x^{*}\right) \leq f(x), \quad \text { for all } x \in C .
$$

Now we show our main results.

Theorem 2.2 Let $K$ be a weakly compact convex subsets of a Banach space $E$ and $T: K \rightarrow$ $K$ be a $\psi$-firmly nonexpansive mapping. Then $T$ has a fixed point, i.e. $F(T) \neq \emptyset$.

Proof Since $K$ is bounded and convex, it is well known (even for nonexpansive mappings) that there exists a sequence $\left\{x_{n}\right\}$ in $K$ such that

$$
\lim _{n \rightarrow \infty}\left\|x_{n}-T x_{n}\right\|=0 .
$$

Let a real valued function $\varphi$ be defined on $K$ by

$$
\varphi(y)=\limsup _{n \rightarrow \infty}\left\|x_{n}-y\right\|^{2}, \quad \forall y \in K .
$$

Then $\varphi$ is convex and continuous, and hence weakly lower semi-continuous (see [25, p.12, Proposition 5]). It follows from Lemma 2.1 that there exists $x^{*} \in K$ such that

$$
\varphi\left(x^{*}\right) \leq \varphi(y) \quad \text { for all } y \in K .
$$


In particular,

$$
\varphi\left(x^{*}\right) \leq \varphi\left(T x^{*}\right) .
$$

Next, we show that $x^{*}=T x^{*}$. It is immediate from (2.5) that

$$
\lim _{n \rightarrow \infty}\left\|\left(x_{n}-T x_{n}\right)-\left(x^{*}-T x^{*}\right)\right\|=\left\|x^{*}-T x^{*}\right\|,
$$

and hence,

$$
\lim _{n \rightarrow \infty} \psi\left(\left\|\left(x_{n}-T x_{n}\right)-\left(x^{*}-T x^{*}\right)\right\|\right)=\psi\left(\left\|x^{*}-T x^{*}\right\|\right) .
$$

Thus, we have

$$
\begin{aligned}
\varphi\left(T x^{*}\right) & =\limsup _{n \rightarrow \infty}\left\|x_{n}-T x^{*}\right\|^{2} \\
& \leq \limsup _{n \rightarrow \infty}\left(\left\|x_{n}-T x_{n}\right\|+\left\|T x_{n}-T x^{*}\right\|\right)^{2} \\
& =\limsup _{n \rightarrow \infty}\left\|T x_{n}-T x^{*}\right\|^{2} \quad(\operatorname{using}(2.5)) \\
& \leq \limsup _{n \rightarrow \infty}\left(\left\|x_{n}-x^{*}\right\|^{2}-\psi\left(\left\|\left(x_{n}-T x_{n}\right)-\left(x^{*}-T x^{*}\right)\right\|\right)\right) \\
& \leq \limsup _{n \rightarrow \infty}\left\|x_{n}-x^{*}\right\|^{2}-\psi\left(\left\|x^{*}-T x^{*}\right\|\right) \quad(\text { using (2.7)) } \\
& =\varphi\left(x^{*}\right)-\psi\left(\left\|x^{*}-T x^{*}\right\|\right) .
\end{aligned}
$$

Therefore together with (2.6), we have

$$
\psi\left(\left\|x^{*}-T x^{*}\right\|\right) \leq \varphi\left(x^{*}\right)-\varphi\left(T x^{*}\right) \leq 0,
$$

and so $x^{*}=T x^{*}$ by the property of $\psi$. This yields the desired conclusion.

Obviously, we also have the following.

Theorem 2.3 Let $K$ be a nonempty bounded closed convex subset of a reflexive Banach space $E$ and $T: K \rightarrow K$ be a $\psi$-firmly nonexpansive mapping. Then $T$ has a fixed point, i.e. $F(T) \neq \emptyset$.

Now we show that the firmly nonexpansive mapping is a subclass of $\psi$-firmly nonexpansive mapping in uniformly convex Banach space.

Lemma 2.4 (Xu [26, Theorem 2]) Let $q>1$ and $M>0$ be two fixed real numbers. Then a Banach space is uniformly convex if and only if there exists a continuous strictly increasing convex function $g:[0,+\infty) \rightarrow[0,+\infty)$ with $g(0)=0$ such that

$$
\|\lambda x+(1-\lambda) y\|^{q} \leq \lambda\|x\|^{q}+(1-\lambda)\|y\|^{q}-\omega_{q}(\lambda) g(\|x-y\|),
$$

for all $x, y \in B_{M}(0)=\{x \in E ;\|x\| \leq M\}$ and $\lambda \in[0,1]$, where $\omega_{q}(\lambda)=\lambda^{q}(1-\lambda)+\lambda(1-\lambda)^{q}$. 
Theorem 2.5 Let $K$ be a nonempty bounded closed convex subset of a uniformly convex Banach space $E$ and $T: K \rightarrow K$ be a firmly nonexpansive mapping. Then $T$ is a $\psi$-firmly nonexpansive mapping.

Proof Since $T$ is firmly nonexpansive, by (2.1), we have

$$
\|T x-T y\| \leq\left\|\frac{x-y}{2}+\frac{T x-T y}{2}\right\|, \quad \forall x, y \in K .
$$

By Lemma 2.4 $\left(q=2, \lambda=\frac{1}{2}\right)$, we obtain

$$
\begin{aligned}
\|T x-T y\|^{2} & \leq\left\|\frac{x-y}{2}+\frac{T x-T y}{2}\right\|^{2} \\
& \leq \frac{1}{2}\|x-y\|^{2}+\frac{1}{2}\|T x-T y\|^{2}-\frac{1}{4} g(\|x-y-(T x-T y)\|) \\
& \leq \frac{1}{2}\|x-y\|^{2}+\frac{1}{2}\|x-y\|^{2}-\frac{1}{4} g(\|x-T x-(y-T y)\|) \\
& =\|x-y\|^{2}-\frac{1}{4} g(\|x-T x-(y-T y)\|) .
\end{aligned}
$$

Let $\psi(s)=\frac{1}{4} g(s)$ for all $s \in[0, \infty)$. The desired result is reached.

Let $A: D(A) \subset E \rightarrow 2^{E}$ be an accretive operator. Let $J_{r}^{A}=(I+r A)^{-1}$, the resolvent of $A$. It is well known that $J_{r}^{A}: R(I+r A) \rightarrow D(A)$ is nonexpansive, where $R(I+r A)$ is range of $(I+r A)$ and $I$ is an identity operator of $E$. Furthermore, for $r>0$ and $t>0$ and $x \in E$,

$$
J_{r}^{A} x=J_{t}^{A}\left(\frac{t}{r} x+\left(1-\frac{t}{r}\right) J_{r}^{A} x\right)
$$

which is referred to as the Resolvent Identity. Now we show that for each $r>0$, the resolvent of $A$ is an $\psi$-firmly nonexpansive mapping also.

Example 2.6 Let $E$ be a uniformly convex Banach space and $A: D(A) \subset E \rightarrow 2^{E}$ be an accretive operator. Then for each $r>0, J_{r}^{A}$ is a $\psi$-firmly nonexpansive mapping defined on $R(I+r A) \cap B_{M}(0)(M>0)$.

Proof It follows from the Resolvent Identity (2.9) that

$$
J_{r}^{A} x=J_{\frac{r}{2}}^{A}\left(\frac{1}{2} x+\frac{1}{2} J_{r}^{A} x\right)
$$

Then we have

$$
\left\|J_{r}^{A} x-J_{r}^{A} y\right\| \leq\left\|J_{r}^{A}\left(\frac{1}{2} x+\frac{1}{2} J_{r}^{A} x\right)-J_{\frac{r}{2}}^{A}\left(\frac{1}{2} y+\frac{1}{2} J_{r}^{A} y\right)\right\| \leq\left\|\frac{1}{2}(x-y)+\frac{1}{2}\left(J_{r}^{A} x-J_{r}^{A} y\right)\right\| .
$$

Using the same proof techniques as Theorem 2.5, we also have

$$
\left\|J_{r}^{A} x-J_{r}^{A} y\right\|^{2} \leq\left\|x_{n}-y\right\|^{2}-\psi\left(\left\|x-J_{r}^{A} x-\left(y-J_{r}^{A} y\right)\right\|\right),
$$

where $\psi=\frac{1}{4} g$. 
The other three similar mappings were introduced by Aoyama et al. [27]. For a subset $C$ of a smooth Banach space $E$, a mapping $T: C \rightarrow E$ is of

(i) type (P) (or firmly nonexpansive-like) if

$$
\langle T x-T y, J(x-T x)-J(y-T y)\rangle \geq 0 \quad \forall x, y \in C,
$$

(ii) type (Q) (or firmly nonexpansive type; see Kohsaka et al. [28]) if

$$
\langle T x-T y,(J x-J T x)-(J y-J T y)\rangle \geq 0 \quad \forall x, y \in C,
$$

(iii) type (R) (or firmly generalized nonexpansive) if

$$
\langle(x-T x)-(y-T y), J T x-J T y\rangle \geq 0 \quad \forall x, y \in C,
$$

where $J$ is the normalized duality mapping of $E$ and $\langle\cdot, \cdot\rangle$ is generalized dual pairs on $E \times E^{*}$.

Remark 2.7 The common point between $\psi$-firmly nonexpansive mapping and the above three mappings is that they all include a firmly nonexpansive mapping in Hilbert spaces as a special case. However, in a uniformly convex Banach space, each firmly nonexpansive mapping is a $\psi$-firmly nonexpansive mapping, but it is not one of the above three mappings since a uniformly convex Banach space may not be smooth.

Remark 2.8 In the framework of a smooth, strictly convex and reflexive Banach space, the fixed point properties of the above three mappings were studied by Aoyama et al. [27], Kohsaka et al. [28] and many mathematical workers. Only in reflexive Banach space, we can obtain the fixed point property of $\psi$-firmly nonexpansive mappings.

\section{Approximation methods of $\psi$-firmly nonexpansive mappings}

We discuss the weak convergence of Picard iteration for $\psi$-firmly nonexpansive mapping.

Lemma 3.1 Let $K$ be a nonempty closed convex subset of a Banach space $E$ and $T: K \rightarrow K$ be $\psi$-firmly nonexpansive with $F(T) \neq \emptyset$. If for any given $x \in K,\left\{x_{n}\right\}$ is defined by Picard iteration sequence

$$
x_{n+1}=T x_{n}=T^{2} x_{n-1}=\cdots=T^{n} x .
$$

Then $\left\{x_{n}\right\}$ is an asymptotic fixed point sequence of $T$, i.e.

$$
\lim _{n \rightarrow \infty}\left\|x_{n}-T x_{n}\right\|=0
$$

Proof Take $p \in F(T)$. Then

$$
\left\|x_{n+1}-p\right\|^{2}=\left\|T x_{n}-p\right\|^{2} \leq\left\|x_{n}-p\right\|^{2}-\psi\left(\left\|x_{n}-T x_{n}\right\|\right) .
$$

Therefore, we have

$$
\left\|x_{n+1}-p\right\|^{2} \leq\left\|x_{n}-p\right\|^{2} \leq \cdots \leq\|x-p\|^{2}
$$


and

$$
\psi\left(\left\|x_{n}-T x_{n}\right\|\right) \leq\left\|x_{n}-p\right\|^{2}-\left\|x_{n+1}-p\right\|^{2} .
$$

Consequently, $\left\{\left\|x_{n}-p\right\|\right\}$ is non-increasing and bounded, and hence the limit $\lim _{n \rightarrow \infty} \| x_{n}-$ $p \|$ exists. So, $\left\{x_{n}\right\}$ is bounded also. It follows from (3.2) that

$$
\lim _{n \rightarrow \infty} \psi\left(\left\|x_{n}-T x_{n}\right\|\right)=0
$$

and hence, by the property of $\psi$. The desired result is obtained.

A Banach space $E$ is said to satisfy Opial's condition [29] if, for any sequence $\left\{x_{n}\right\}$ in $E$, $x_{n} \rightarrow x$ implies

$$
\limsup _{n \rightarrow \infty}\left\|x_{n}-x\right\|<\limsup _{n \rightarrow \infty}\left\|x_{n}-y\right\|, \quad \forall y \in E \text { with } x \neq y .
$$

In particular, Opial's condition is independent of uniformly convex (smooth) since the $l^{p}$ spaces satisfy this condition for $1<p<\infty$ while it fails for the $L^{p}(p \neq 2)$ spaces. In fact, spaces satisfying Opial's condition need not even by isomorphic to uniformly convex spaces [30].

Theorem 3.2 Let $K$ be a weakly compact convex subset of a Banach space E satisfying Opial's condition and $T: K \rightarrow K$ be firmly type nonexpansive. Then for any given $x \in K$, $\left\{x_{n}\right\}$, defined by Picard iteration (3.1) weakly converges to some fixed point of $T$.

Proof It follows from Theorem 2.2 that $F(T) \neq \emptyset$. Then following Lemma 3.1, we see that $\left\{x_{n}\right\}$ is bounded, the limit $\lim _{n \rightarrow \infty}\left\|x_{n}-p\right\|$ exists for each $p \in F(T)$ and

$$
\lim _{n \rightarrow \infty}\left\|x_{n}-T x_{n}\right\|=0 .
$$

The weak compactness of $K$ means that there exists a subsequence $\left\{x_{n_{k}}\right\}$ of $\left\{x_{n}\right\}$ such that $\left\{x_{n_{k}}\right\}$ weakly converges to some point of $K$, say $x^{*}$. Then using the proof technique of Theorem 2.2, we have $x^{*}=T x^{*}$ since by Opial's condition,

$$
\limsup _{k \rightarrow \infty}\left\|x_{n_{k}}-x^{*}\right\| \leq \limsup _{k \rightarrow \infty}\left\|x_{n_{k}}-x\right\| \quad \text { for all } x \in K
$$

Next we show that $\left\{x_{n}\right\}$ weakly converges to $x^{*}$. Let $y$ is another weak limit point of $\left\{x_{n}\right\}$ and $x^{*} \neq y$. Then we can choose a subsequence $\left\{x_{n_{j}}\right\}$ that weakly converges to $y$, and hence $y=T y$. Since $\lim _{n \rightarrow \infty}\left\|x_{n}-p\right\|$ exists for each $p \in F(T)$, we have

$$
\begin{aligned}
\lim _{n \rightarrow \infty}\left\|x_{n}-x^{*}\right\| & =\limsup _{k \rightarrow \infty}\left\|x_{n_{k}}-x^{*}\right\| \\
& <\limsup _{k \rightarrow \infty}\left\|x_{n_{k}}-y\right\|=\lim _{n \rightarrow \infty}\left\|x_{n}-y\right\| \\
& =\limsup _{j \rightarrow \infty}\left\|x_{n_{j}}-y\right\|<\limsup _{j \rightarrow \infty}\left\|x_{n_{j}}-x^{*}\right\|=\lim _{n \rightarrow \infty}\left\|x_{n}-x^{*}\right\|,
\end{aligned}
$$

a contradiction, and hence $x^{*}=y$. 
Similarly, we also have the following.

Theorem 3.3 Let $K$ be a nonempty closed convex subset of a reflexive Banach space $E$ satisfying Opial's condition and $T: K \rightarrow K$ be firmly type nonexpansive with $F(T) \neq \emptyset$. Then for any given $x \in K,\left\{x_{n}\right\}$, defined by Picard iteration (3.1) weakly converges to some fixed point of $T$.

Remark 3.4 Theorem 3.2 is applicable to $l^{p}(1<p<\infty)$ and $L^{2}$. However, we do not know whether it works in $L^{p}$ for $0<p<2$ and $2<p<\infty$.

Recall a Banach space $E$ is said to have (i) a Gâteaux differentiable norm (we also say that $E$ is smooth), if the limit

$$
D_{x}(y)=\lim _{t \rightarrow 0} \frac{\|x+t y\|-\|x\|}{t}
$$

exists for each $x(x \neq 0), y \in E$; (ii) a uniformly Gâteaux differentiable norm, if for each $y$ in $E$, the limit $D_{x}(y)$ is uniformly attained for bounded $0 \neq x \in E$; (iii) a Fréchet differentiable norm, if for each $x \in E, x \neq 0$, the limit $D_{x}(y)$ is attained uniformly for bounded $y \in E$.

The value of $x^{*} \in E^{*}$ at $y \in E$ is denoted by $\langle y, x\rangle$, and the normalized duality mapping from $E$ into $2^{E^{*}}$ is denoted by $J$, that is,

$$
J(x)=\left\{f \in E^{*}:\langle x, f\rangle=\|x\|\|f\|,\|x\|=\|f\|\right\}, \quad \forall x \in E .
$$

It is well known (see Brower [31, p.44]) that for a smooth Banch space $E$, the normalized duality mapping $J$ is single-valued, and, moreover,

$$
D_{x}(y)=\frac{\langle y, J(x)\rangle}{\|x\|} .
$$

A Banach space $E$ is said to be (iv) strictly convex if $\|x\|=\|y\|=1, x \neq y$ implies $\frac{\|x+y\|}{2}<1$; (v) uniformly convex if for all $\varepsilon \in[0,2], \exists \delta_{\varepsilon}>0$ such that $\|x\|=\|y\|=1$ implies $\frac{\|x+y\|}{2}<1-\delta_{\varepsilon}$ whenever $\|x-y\| \geq \varepsilon$.

In 1979, Bruck [20] explicitly introduced the following concept. Let $\Gamma$ denote the set of strictly increasing convex functions $\gamma: \mathbb{R}^{+} \rightarrow \mathbb{R}^{+}$with $\gamma(0)=0$. A mapping $T$ is said to be of type $\Gamma$ if there exists $\gamma \in \Gamma$ such that for all $x, y \in D(T)$ and $c \in[0,1]$

$$
\gamma(\|c T x+(1-c) T y-T(c x+(1-c) y)\|) \leq\|x-y\|-\|T x-T y\| .
$$

Three facts about such mappings are easy to observe. Mappings of type $\Gamma$ are nonexpansive; affine nonexpansive mappings are of type $\Gamma$; Mappings of type $\Gamma$ have convex fixed point sets. Bruck $[20,21]$ showed that each nonexpansive mapping is of type $\Gamma$ in a uniformly convex Banach space. See also [7, Proposition 10.3] for a proof.

Next we give the weak convergence of Picard iteration in a uniformly convex Banach space by using the proof technique developed in Reich [19, 32].

Theorem 3.5 Let E be a uniformly convex Banach space with a Fréchet differentiable norm and $K$ be a nonempty closed convex subset of $E$. If $T: K \rightarrow K$ is $\psi$-firmly nonexpansive with 
$F(T) \neq \emptyset$, then for any given $x \in K,\left\{x_{n}\right\}$, defined by Picard iteration (3.1) weakly converges to some fixed point of $T$.

Proof It follows from Lemma 3.1 that $\left\{x_{n}\right\}$ is bounded, the limit $\lim _{n \rightarrow \infty}\left\|x_{n}-y\right\|$ exists for each $y \in F(T)$ and

$$
\lim _{n \rightarrow \infty}\left\|x_{n}-T x_{n}\right\|=0
$$

Then $\left\{x_{n}\right\}$ is weakly compact. Similarly to the proof of Theorem 3.2, we only need show that $\left\{x_{n}\right\}$ has unique weak limit point. Let $p$ and $q$ are two weak limit points of $\left\{x_{n}\right\}$. Then Browder Demiclosedness Principle [33] means that $p, q \in F(T)$. Thus both the limits $\lim _{n \rightarrow \infty}\left\|x_{n}-p\right\|$ and $\lim _{n \rightarrow \infty}\left\|x_{n}-q\right\|$ exist. Then the remainder of the proof is identical to the proof of Theorem 10.6 in Reference [7, pp.114-115] with the help of the mappings of type $\Gamma$. Which is a repeat works, we omit it.

By Theorem 2.5, the following corollary about firmly nonexpansive mappings is obvious.

Corollary 3.6 Let E be a uniformly convex Banach space with a Fréchet differentiable norm and $K$ be a nonempty closed convex subset of $E$. If $T: K \rightarrow K$ is firmly nonexpansive with $F(T) \neq \emptyset$, then for any given $x \in K,\left\{x_{n}\right\}$, defined by Picard iteration (3.1) weakly converges to some fixed point of $T$.

Remark 3.7 Theorem 3.5 is dependent of Theorem 3.2 or 3.3 since the $l^{p}$ spaces satisfy Opial's condition for $1<p<\infty$ while it fails for the $L^{p}(p \neq 2)$ spaces. On the other hand, spaces satisfying Opial's condition need not even by isomorphic to uniformly convex spaces [30].

\section{Competing interests}

The authors declare that they have no competing interests.

\section{Authors' contributions}

The work presented here was carried out in collaboration between all authors. All authors contributed equally and significantly to writing this manuscript. All authors have contributed to, seen and approved the final manuscript.

\section{Acknowledgements}

The authors would like to thank the editor and the anonymous referee for useful comments and valuable suggestions on the language and structure of our manuscript. This work is partially supported by the National Natural Science Foundation of P.R. China (Grant Nos. 11171094, 11271112) and partially done when he was visiting The Hong Kong Polytechnic University.

Received: 9 January 2014 Accepted: 14 March 2014 Published: 26 Mar 2014

\section{References}

1. Reich, S: The fixed point property for nonexpansive mappings. I. Am. Math. Mon. 83, 266-268 (1976)

2. Reich, S: The fixed point property for nonexpansive mappings. II. Am. Math. Mon. 87, 292-294 (1980)

3. Sadovski, VN: Application of topological methods in the theory of periodic solutions of nonlinear differential-operator equations of neutral type. Dokl. Akad. Nauk SSSR 200, 1037-1040 (1971) (in Russian). Sov. Phys. Dokl. 12 (1971)

4. Alspach, DE: A fixed point free nonexpansive map. Proc. Am. Math. Soc. 82, 423-424 (1981)

5. Istrăţescu, VI: Fixed Point Theory: An Introduction. Reidel, Dordrecht (1981)

6. Kirk, WA, Sims, B: Examples of fixed point free mappings. In: Handbook of Metric Fixed Point Theory, pp. 35-91. Kluwer Academic, Dordrecht (2001)

7. Goebel, K, Kirk, WA: Topics in Metric Fixed Point Theory. Cambridge Stud. Adv. Math., vol. 28. Cambridge University Press, Cambridge (1990)

8. Browder, FE: Non-expansive nonlinear operators in Banach spaces. Proc. Natl. Acad. Sci. USA 54, 1041-1044 (1965)

9. Göhde, D: Zum prinzip der kontraktiven abbildung. Math. Nachr. 30, 251-258 (1965)

10. Kirk, WA: A fixed point theorem for mappings which do not increase distances. Am. Math. Mon. 72, 1004-1006 (1965) 
11. Sims, B, Smyth, MA: On some Banach space properties sufficient for weak normal structure and their permanence properties. Trans. Am. Math. Soc. 351, 497-513 (1999)

12. Baillon, JB, Schöneberg, R: Asymptotic normal structure and fixed points of nonexpansive mappings. Proc. Am. Math. Soc. 81, 257-264 (1981)

13. Maurey, B: Points fixes des contractions sur un convex fermé de $L^{1}$. In: Seminaire d'Analyse Fonctionelle, $1980-1981$. École Polytechnique, Palaiseau (1981)

14. Dowling, PN, Lennard, CJ, Turett, B: Weak compactness is equivalent to the fixed point property in $c_{0}$. Proc. Am. Math. Soc. 132, 1659-1666 (2004)

15. Domínguez Benavides, T, Pineda, MAJ: Fixed points of nonexpansive mappings in spaces of continuous functions Proc. Am. Math. Soc. 133, 3037-3046 (2005)

16. Domínguez Benavides, T, Pineda, MAJ, Prus, S: Weak compactness and fixed point property for affine mappings. J. Funct. Anal. 209, 1-15 (2004)

17. Aksoy, A, Khamsi, MA: Nonstandard Methods in Fixed Point Theory. Springer, Berlin (1990)

18. Elton, J, Lin, P-K, Odell, E, Szarek, S: Remarks on fixed point problem for nonexpansive mappings. In: Sine, R (ed.) Fixed Points and Nonexpansive Maps. Contemporary Math., vol. 18, pp. 87-120 (1983)

19. Reich, S: Weak convergence theorems for nonexpansive mappings in Banach spaces. J. Math. Anal. Appl. 67, 274-276 (1979)

20. Bruck, RE: A simple proof of the mean ergodic theorem for nonlinear contractions in Banach spaces. Isr. J. Math. 32, 107-116 (1979)

21. Bruck, RE: On the convex approximation property and the asymptotic behavior of nonlinear contractions in Banach spaces. Isr. J. Math. 38, 304-314 (1981)

22. Baillon, JB: Un théorème de type ergodique pour les contractions non linéairs dans un espaces de Hilbert. C. R. Acad. Sci. Paris Sér. A-B 280, 1511-1541 (1975)

23. Bruck, RE: Nonexpansive projections on subsets of Banach spaces. Pac. J. Math. 47, 341-355 (1973)

24. Song, Y, Chai, X: Halpern iteration for firmly type nonexpansive mappings. Nonlinear Anal. 71(10), $4500-4506$ (2009)

25. Aubin, JP, Ekeland, I: Applied Nonlinear Analysis. A Wiley-Interscience Publication. Wiley, New York (1984)

26. Xu, HK: Inequality in Banach spaces with applications. Nonlinear Anal. 16, 1127-1138 (1991)

27. Aoyama, K, Kohsaka, F, Takahashi, W: Three generalizations of firmly nonexpansive mappings: their relations and continuity properties. J. Nonlinear Convex Anal. 10(1), 131-147 (2009)

28. Kohsaka, F, Takahashi, W: Existence and approximation of fixed points of firmly nonexpansive type mappings in Banach spaces. SIAM J. Optim. 19(2), 824-835 (2008)

29. Opial, Z: Weak convergence of the sequence of successive approximations for nonexpansive mappings in Banach spaces. Bull. Am. Math. Soc. 73, 591-597 (1967)

30. Lami Dozo, E: Multivalued nonexpansive mappings and Opial's condition. Proc. Am. Math. Soc. 38, 286-292 (1973)

31. Browder, FE: Nonlinear operators and nonlinear equations of evolution in Banach spaces. In: Proc. Symp. Pure Math., vol. 18. Part 2. Am. Math. Soc., Providence (1976)

32. Reich, S: Strong convergence theorems for resolvents of accretive operators in Banach spaces. J. Math. Anal. Appl. 75 287-292 (1980)

33. Browder, FE: Semicontractive and semiaccretive nonlinear mappings in a Banach space. Bull. Am. Math. Soc. 74 660-665 (1968)

10.1186/1687-1812-2014-81

Cite this article as: Song and Huang: Fixed point property and approximation of a class of nonexpansive mappings. Fixed Point Theory and Applications 2014, 2014:81

\section{Submit your manuscript to a SpringerOpen ${ }^{\circ}$ journal and benefit from:}

- Convenient online submission

- Rigorous peer review

Immediate publication on acceptance

- Open access: articles freely available online

- High visibility within the field

- Retaining the copyright to your article 